\title{
Fiabilité mécanique : méthode de l'espérance mathématique du rang après censures et méthode des durées de vies fictives pour données fortement censurées
}

\author{
PATRICK LyOnNeT ${ }^{1, a}$ ET SÉBAStien Theuillon ${ }^{2}$ \\ 1 ENISE, LTDS UMR 5513, 58 rue Jean Parot, 42000 St. Étienne, France \\ 2 LSA, Ligeron-Sonovision-Itep, Parc des Algorithmes, Bâtiment Euclide, 91194 St Aubin Cedex, France
}

Reçu le 4 septembre 2002, accepté le 5 fevrier 2004

\begin{abstract}
Résumé - Ce travail fait suite à une première étude publiée en 1992 dans la revue statistique appliquée [P. Lyonnet, J. Fauchon, Comparaison de la méthode d'estimation des rangs dans le cas de données fortement censurées proposée par Johnson et de celle proposée par P. Lyonnet, Revue de Statistique appliquée XXXX (3) (1992) 73-78] et concernant les données censurées que l'on rencontre en milieux industriels. En effet la détermination des modèles de durées de vie des composants mécaniques s'établit à partir de ce type d'échantillon, les méthodes de traitement des données censurées doivent par conséquent être robustes. Une première approche de calcul des rangs après censures venant compléter l'approche faite par Johnson L. [L. Johnson, The statistical treatment of fatigue experiment, Elsevier Publishing Company, 1964] avait été développée par LYONNET P. et validée à partir d'échantillons simulés. Une version plus ergonomique de cette méthode est proposée ici « $\operatorname{MER}_{(2)}$ ", ainsi que la présentation d'une nouvelle approche consistant à remplacer les censures par des durées fictives obtenues à partir du calcul de l'espérance de vie résiduelle « MDF », les résultats sont comparés et analysés.
\end{abstract}

Mots clés : Fiabilité mécanique / censures / loi de Weibull / durées de vie

\begin{abstract}
Mechanical reliability : Expected rank method and fictitious lifetime method for high censored dater. This papers present an prolongation to a published work presented in 1992 [P. Lyonnet, J. Fauchon, Comparaison de la méthode d'estimation des rangs dans le cas de données fortement censurées proposée par Johnson et de celle proposée par P. Lyonnet, Revue de Statistique appliquée XXXX (3) (1992) 73-78] and concerne industrial reliability's problems. In fact, lifetime mechanics models are often obtained with censored samples. For this reason censored traitment have to be reliable. Here a first method employed to arrive at mean order number "MON" for the actual failures in incomplete samples is improved. An other method designed as "lifetimes fictitious method "LFM" is also presented. Both are tested and compared to Johnson' method by simulation.
\end{abstract}

Key words: Mechanical reliability / censured data / Weibull law / lifetimes

\section{Introduction}

L'étude réalisée ici concerne les problèmes d'estimation des paramètres de lois, type loi de Weibull, dans le cas d'échantillons censurés et fortement censurés que l'on rencontre dans les analyses de retours d'expériences en fiabilité. Ce problème ancien a connu différentes propositions de solutions $[3,4]$. Ces solutions ne sont pas toujours satisfaisantes soit du fait de la difficulté de mise en œuvre, soit au niveau des résultats obtenus. En mécanique la loi de Weibull est particulièrement appréciée, la connaissance

\footnotetext{
a Auteur correspondant : lyonnet@enise.fr
}

du paramètre de forme est d'une grande utilité pour la prise de décision (par exemple en maintenance), l'obtention d'une valeur correcte de ce paramètre s'appuie sur une bonne modélisation et la résolution du problème des données incomplètes. Deux approches sont développées et présentées ici.

\section{Rappels de statistique et de fiabilité}

$R(t)=$ Fonction fiabilité qui représente la probabilité de fonctionnement à l'instant $t$ 
$F(t)=$ Fonction cumulée de défaillances

$$
F(t)+R(t)=1
$$

Loi de Weibull :

$R(t)=e^{-\left(\frac{t-\gamma}{\eta}\right)^{\beta}} \gamma, \eta, \beta$ : paramètres de la loi de Weibull $E(r)=\sum_{i} r_{i} P\left(r_{i}\right)$ : Espérance mathématique pour une loi discrète, ( $r$ étant ici le rang)

Il existe plusieurs façons d'obtenir les paramètres de la loi de Weibull à partir d'un échantillon de données de durées de vie, on peut classiquement citer :

- La méthode des quantiles,

- La méthode des moments,

- La méthode du maximum de vraisemblance,

- La méthode du graphique d'Allan Plait

Cette dernière est très utilisée dans le milieu industriel et permet l'estimation du paramètre de décalage $« \gamma »$. Elle se détermine avec l'estimation de la fonction cumulée de défaillances « $F(t)$ » établie à partir de l'échantillon ordonné. Pour cela, il faut déterminer la fonction de répartition de la $k^{\text {ème }}$ défaillance dans l'échantillon ordonné de taille « $n »$, qui est défini ci-après.

$$
\left\{t_{1}, t_{2}, t_{3 .} t_{k, \ldots \ldots \ldots \ldots \ldots \ldots . . .} t_{n}\right\}
$$

On établit aisément que la loi de réalisation de la variable aléatoire « $K »$ obéit à une loi binomiale de paramètre $\ll p »$, tel que :

$p=F(t)$, ainsi

$$
P(K=k)=C_{n}^{k}\left[F\left(t_{k}\right)\right]^{k}\left[1-F\left(t_{k}\right)\right]^{n-k}
$$

et par la suite

$$
F_{t k}\left(t_{k}\right)=\sum_{i=k}^{i=n} C_{n}^{i}\left[F\left(t_{k}\right)\right]^{i}\left[1-F\left(t_{k}\right)\right]^{n-i}
$$

un critère d'optimisation généralement utilisé est de déterminer $F_{t k}\left(t_{k}\right)=0,5$ ce qui conduit à :

$$
\sum_{i=k}^{i=n} C_{n}^{i}\left[F\left(t_{k}\right)\right]^{i}\left[1-F\left(t_{k}\right)\right]^{n-i}=0,5
$$

Une bonne approche de la résolution de cette équation s'obtient par un calcul connu sous le nom de méthode des rangs médians résumé par la formule suivante :

$$
F(t)=\frac{m i-0,3}{n+0,4}
$$

$n=$ nombre d'éléments observés défaillants plus les censures; « $m_{i} »=$ rang de la défaillance survenant à l'instant $t$ (c'est l'élément que nous nous proposons de calculer, qui est parfois appelé rang corrigé ou espérance mathématique du rang après censures).

\section{Position du problème d'estimation de $« \boldsymbol{F}(\boldsymbol{t}) »$ avec des censures aléatoires à droite}

\section{Hypothèses :}

- On ne prend en compte que les $1^{\text {res }}$ défaillances.

- Le matériel appartient à la même famille, population homogène

Après classement des défaillances par ordre croissant, on s'aperçoit que l'on a des éléments qui ne sont pas tombés en panne et qui ne totalisent pas un nombre d'heures de fonctionnement aussi grand que le dernier défaillant. On se trouve donc avec une configuration des éléments classés par ordre croissant qui a l'allure suivante :

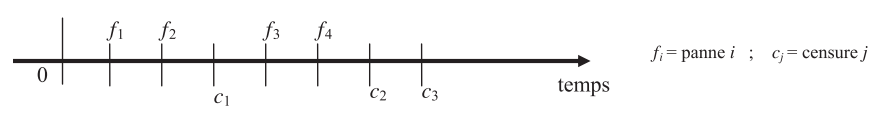

Les éléments censurés ne sont pas pris en compte, mais viennent modifier le rang des défaillants qui se trouvent après eux. On est amené à se poser la question : comment sont modifiés les rangs des défaillants après censure?

\section{Méthode proposée par JOHNSON L. connue sous le nom de Formule de Johnson $\ll \mathbf{F J} »,[2]$}

Pour les deux premiers, le rang est déterminé sans difficulté; c'est un rang certain, ici 1 et 2 . Par contre, pour les $3^{\mathrm{e}}$ et $4^{\mathrm{e}}$ défaillances, on peut dire que cela dépend de la fin de durée de vie de l'élément censuré. En effet nous constatons que :

$\ll c_{1} »$ peut être défaillant entre 2 et 3 (événement a)

$\ll c_{1} »$ peut être défaillant entre 3 et 4 (événement b)

$\ll c_{1} »$ peut être défaillant après 4 (événement $\mathrm{c}$ )

\section{Hypothèse de Johnson (interprétation probabiliste)}

On considère que ces trois éventualités sont équiprobables [2]. De ce fait, il en découle que :

- Le rang « $3 »$ peut être donné à la défaillance « $3 »$ avec une probabilité de :

$$
P\left(3^{\mathrm{e}} \text { déf. ait le rang } « 3 »\right)=P(b)+P(c)=2 / 3
$$

Parce que : $P(a)+P(b)+P(c)=1$ d'où $P(a)=P(b)=$ $P(c)=1 / 3$, (équiprobabilité)

- le rang « 4 » peut être donné à la $3^{\mathrm{e}}$ défaillance avec une probabilité de :

$$
P\left(3^{\mathrm{e}} \text { défaillance ait le rang } 4\right)=P(a)=1 / 3
$$

Ainsi, si on veut estimer l'Ordre Moyen « OMi» de la défaillance $\ll \mathrm{i} »$ on a : 
- Ordre Moyen $3^{\mathrm{e}}$ défaillance :

$$
O M_{3}=3,2 / 3+4,1 / 3=3,33
$$

On fera le même type de démarche pour les défaillants suivants, ainsi :

$$
\mathrm{OM}_{4}=4,1 / 3+5 \times 2 / 3=4,66
$$

Cette approche, conduit à modifier le rang de la défaillance, lorsqu'il y a des censures avant, ce qui se résume par une formule pratique connue sous le nom de Formule de Johnson «FJ» qui permet de calculer l'incrément «I » à donner au rang suivant lorsque l'on se trouve dans cette situation, ainsi :

\section{voir l'équation ci-dessus}

Cette méthode ne tient pas compte de la distance séparant le suspendu du défaillant suivant, pour estimer l'incrément à donner au rang. Et elle ne tient pas compte de la forme de la distribution des défaillances pour estimer les rangs après suspension. Ceci nous conduit à proposer quelques améliorations à la méthode de calcul de la fonction de répartition dans le cas de données censurées.

\section{Estimation du rang après censure s'appuyant sur la méthode l'espérance mathématique du rang "MER $\mathrm{M}_{(1)}$ », proposée par LYONNET P. [1]}

Cette méthode tient compte de l'âge de la censure et aussi de la loi de distribution des défaillances. Pour cela il est nécessaire de procéder en deux grandes étapes, la première estimant les premiers rangs après censure à partir d'un modèle de densité de probabilité de défaillances ne nécessitant pas le calcul de paramètres (modèle non paramétrique), par exemple une loi uniforme convient. La deuxième étape reprend le calcul des rangs à partir du premier modèle obtenu, cette opération pouvant être réitérée plusieurs fois.

\section{Première étape : modélisation selon une loi uniforme :}

\section{Hypothèses :}

- Identiques à celles de Johnson avec en plus, la détermination de la probabilité qu'un élément censuré à $c_{j}$ tombe en panne à $t$ (avec $t>c_{j}$ ) proportionnelle à la différence $\left(t-c_{j}\right)$.

- Les censurés ne sont pas confondus avec les défaillances.

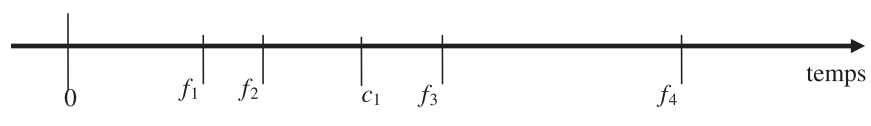

Pour le $3^{\text {e }}$ défaillant, le rang n'est pas déterminé car il y a eu une censure, ainsi :

Prob. $\left(3^{\text {e }}\right.$ déf. ait le rang 3$)=P\left(c_{1}\right.$ soit déf. après $\left.f_{3}\right)=$ $P\left(c_{1}>f_{3}\right)$

Si on fait l'hypothèse que la défaillance de l'élément censuré obéit à une loi uniforme sur $\left[c_{1}, f_{4}\right]$, alors :

$$
\text { Prob. }\left(3^{\text {e }} \text { déf. ait le rang } 3\right)=\frac{f_{4}-f_{3}}{f_{4}-c_{1}}
$$

de même :

$$
\text { Prob.( } \left.3^{\text {e }} \text { déf. ait le rang } 4\right)=\frac{f_{3}-c_{1}}{f_{4}-c_{1}}
$$

Et ainsi de suite on en déduit le rang des défaillances après censures par le calcul de l'espérance mathématique du rang, tel que :

$$
M E R_{(1)}=E(r)=\sum_{i} r_{i} P\left(r_{i}\right)
$$

\section{Remarque sur la méthode $\mathrm{MER}_{(1)}$}

D'une part, cette méthode tient compte des temps séparant deux défaillances consécutives, en effet si cette distance est grande, la probabilité qu'une défaillance des éléments censurés apparaisse dans cet intervalle est grande. En ce sens, elle paraît plus performante que la méthode de Johnson. D'autre part, le modèle utilisé précédemment dans le calcul des probabilités des défaillances pour les éléments censurés est une loi uniforme ce qui offre l'avantage d'être calculable sans recherche de paramètre de loi.

Deuxième étape : amélioration dans une seconde phase avec le modèle de Weibull

Une meilleure estimation de la modélisation de la probabilité de défaillance des éléments censurés consiste à prendre pour modèle une loi s'approchant le plus possible de la réalité loi de Weibull $(\eta, \beta, \gamma)$ par exemple, au lieu de la loi uniforme. Le calcul des rangs précédents nous permet de trouver un premier modèle de Weibull $\left(W_{1}\right)$, qui sera utilisé pour déterminer les probabilités de défaillances après censure. Ceci s'exprime de la façon suivante :

$$
P\left(3^{\mathrm{e}} \text { déf. ait le rang } 3\right)=\frac{R\left(f_{3}\right)-R\left(f_{4}\right)}{R\left(c_{1}\right)-R\left(f_{4}\right)}
$$


de même :

$$
P\left(3^{\text {e }} \text { def. ait le rang } 4\right)=\frac{R\left(c_{1}\right)-R\left(f_{3}\right)}{R\left(c_{1}\right)-R\left(f_{4}\right)}
$$

Il est nécessaire de connaître les paramètres $\beta, \gamma, \eta$ que nous sommes justement en train de chercher. La méthode proposée devra donc tenir compte de ce problème, et procéder par itération.

$1^{\mathrm{rs}}$ algorithmes de la méthode «EMR $R_{(1)}$, recherche des rangs dans le cas des censures aléatoires à droites en utilisant une itération, loi uniforme/loi de Weibull

$1^{\text {re }}$ étape

Dans un premier temps, on veut modéliser une loi approximant la loi de défaillances. On utilise, donc, pour estimer les rangs des éléments après censures, une distribution uniforme jusqu'au dernier élément fonctionnant, ou la méthode de Johnson.

$2^{e}$ étape

On détermine la fonction de répartition puis les paramètres $\eta_{1}, \beta_{1}, \gamma_{1}$.

\section{$3^{e}$ étape}

La loi de défaillances $F(t)=1-R(t)$ nous permet de déterminer la probabilité de défaillance des éléments censurés, et ainsi de mieux estimer l'ordre des défaillances.

$$
F(t)=1-\mathrm{e}^{-\left(\left(t-\gamma_{1}\right) / \eta_{1}\right)^{\beta_{1}}}
$$

\section{$4^{e}$ étape}

On utilise ces nouveaux rangs pour déterminer la nouvelle loi.

Il faut remarquer que lorsque nous avons un nombre important de censures, il faut envisager toutes les combinaisons de défaillances après censures, ce qui est difficile, voir le schéma ci-après. Une simplification est proposée dans le deuxième algorithme, $\operatorname{EMR}_{(2)}$

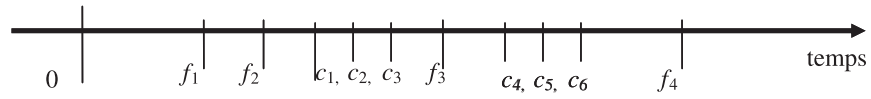

$2^{e}$ algorithme de la méthode proposé par P. LYONNET «EMR (2) *: recherche des rangs dans le cas des censures multiples en utilisant une itération loi uniforme/loi de Weibull

Dans ce deuxième algorithme $[5,6]$ on remplace l'ensemble des éléments censurés avant le calcul du rang modifié de la défaillance par une valeur moyenne représentative des censures et de taille « $n_{\mathrm{c}} »$, ce qui conduit au schéma suivant et au calcul du rang qui en découle :

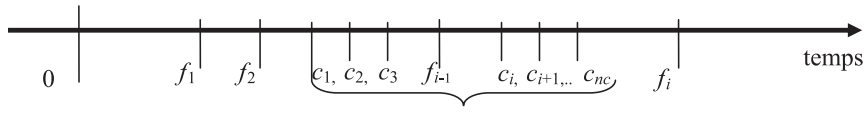

$$
\bar{c} ; n_{\mathrm{c}},(n)
$$

$n_{\mathrm{c}}=$ le nombre d'éléments censurés avant la défaillance, (on notera « $n »$, lorsqu'il n'y a pas de confusion) et $\bar{c}=$ durée moyenne des censures.

Calcul du rang après censures :

$$
\begin{gathered}
\bar{c}=\frac{\sum_{i=1}^{n_{\mathrm{c}}} c_{i}}{n_{c}} \\
P\left(\bar{c}<t_{i}\right)=p \\
P\left(\bar{c} \geq t_{i}\right)=1-p=q \\
p+q=1
\end{gathered}
$$

avec :

$$
R\left(t_{i}\right)=e^{-\left(\frac{t_{i}-\gamma_{1}}{\eta_{1}}\right)^{\beta_{1}}}
$$

À l'aide la loi binomiale on peut calculer :

$$
\begin{aligned}
P\left(r_{i}+0\right) & =C_{n}^{0} p^{0} q^{n} \\
P\left(r_{i}+1\right) & =C_{n}^{1} p^{1} q^{n-1} \\
P\left(r_{i}+n_{c}\right) & =C_{n}^{n} p^{n} q^{0}
\end{aligned}
$$

Le calcul du rang moyen après censures devient :

$$
\begin{gathered}
E M R_{(2)}=\left(r_{i}+0\right) P\left(r_{i}+0\right)+\left(r_{i}+1\right) P\left(r_{i}+1\right) \\
+\left(r_{i}+2\right) P\left(r_{i}+2\right) P\left(r_{i}+2\right)+\ldots(r i+j) P\left(r_{i}+j\right) \ldots \\
+\left(r_{i}+n\right) P\left(r_{i}+n\right)
\end{gathered}
$$

Le calcul du rang moyen après censure devient avec cette méthode très aisé.

$$
m_{i}=\operatorname{EMR}_{(2)}=r_{i}+n p
$$

\section{Cas des ex æquo}

Si deux censures sont confondues, leurs prises en compte se fera naturellement à partir de la taille « $n$ » qui intervient dans la loi binomiale.

\section{Méthodes des durées de vie fictives MDF» (remplacement des censures par des durées estimées)}

Le principe de la méthode est de remplacer dans l'échantillon censuré toutes les censures par une durée de vie fictive $« t_{\mathrm{fi}} »$ calculée à partir de la durée de vie résiduelle. Ce temps de survie résiduelle $\ll L(t) »$, s'obtient 
Tableau 1. Résultats comparatifs complets.

\begin{tabular}{|c|c|c|c|c|c|}
\hline $\mathrm{N}^{\circ}$ & Temps & $\begin{array}{l}\text { Défaillant (1) } \\
\text { Censure }(0)\end{array}$ & $\begin{array}{l}F(\mathrm{t}) \text { avec } \ll \mathrm{FJ} », \\
\text { Johnson L. }\end{array}$ & $\begin{array}{l}F(\mathrm{t}) \text { avec } \\
\mathrm{MDF}\end{array}$ & $F(\mathrm{t})$ avec $\ll \operatorname{MER}_{(2)} »$ \\
\hline 1 & 6 & 1 & & & \\
\hline 2 & 6 & 1 & & & \\
\hline 3 & 6 & 1 & 0,126 & 0,126 & 0,126 \\
\hline 4 & 6 & 0 & & $F(t=x)=0,173$ & \\
\hline 5 & 7 & 1 & 0,1754 & 0,219 & 0,177 \\
\hline 6 & 9 & 0 & & $F(t=x)=0,266$ & \\
\hline 7 & 10 & 1 & 0,227 & 0,313 & 0,229 \\
\hline 8 & 10 & 0 & & $F(t=x)=0,359$ & \\
\hline 9 & 11 & 0 & & $F(t=x)=0,406$ & \\
\hline 10 & 13 & 1 & 0,288 & 0,453 & 0,293 \\
\hline 11 & 16 & 1 & 0,348 & 0,500 & 0,355 \\
\hline 12 & 17 & 0 & & $F(t=x)=0,547$ & \\
\hline 13 & 19 & 0 & & $F(t=x)=0,594$ & \\
\hline 14 & 20 & 0 & & $F(t=x)=0,640$ & \\
\hline 15 & 22 & 1 & 0,432 & 0,686 & 0,436 \\
\hline 16 & 23 & 1 & 0,515 & 0,734 & 0,489 \\
\hline 17 & 25 & 0 & & $F(t=x)=0,780$ & \\
\hline 18 & 32 & 0 & & $F(t=x)=0,827$ & \\
\hline 19 & 32 & 0 & & $F(t=x)=0,874$ & \\
\hline 20 & 34 & 0 & & $F(t=x)=0,920$ & \\
\hline 21 & 35 & 0 & & $F(t=x)=0,967$ & \\
\hline
\end{tabular}

à partir du calcul de l'espérance mathématique conditionnelle à $c_{i}$.

$$
L(t)=E\left(t-c_{i} / t>c_{i}\right)=\int_{c_{i}}^{\infty} \frac{R(t) \mathrm{d} t}{R\left(c_{i}\right)}
$$

d'où la durée fictive « $t_{\mathrm{fi}} »$ qui remplacera la censure « $\mathrm{ci}$ »

$$
t_{\mathrm{fi}}=c_{i}+L(t)
$$

6.1 Cas de la loi exponentielle de paramètre « $\lambda$ » ce qui correspond au cas particulier d'une loi de Weibull de paramètre $\ll \beta=1 »$

$$
L(t)=E\left(t-c_{i} / t>c_{i}\right)=\int_{c_{i}}^{\infty} \frac{e^{-\lambda t} \mathrm{~d} t}{e^{-\lambda C i}}=\frac{1}{\lambda}
$$

Résultat attendu qui correspond à un non vieillissement.

\subsection{Cas de la loi de Weibull, $\beta \neq 1$}

$$
L(t)=E\left(t-c_{i} / t>c_{i}\right)=\int_{c_{i}}^{\infty} \frac{e^{-\left(\frac{t-\gamma}{\eta}\right)^{\beta}} \mathrm{d} t}{e^{-\left(\frac{c i-\gamma}{\eta}\right)^{\beta}}}
$$

\section{Cas des ex æquo}

Si nous avons $« n »$ censures $« c_{i} »$ au même instant, le temps fictif de remplacement est de fait le même $« t_{\mathrm{fi}} »$. Le calcul de la fonction de répartition $F\left(t_{\mathrm{fi}}\right)$ prendra en compte cette particularité en affectant un poids « $n$ » égal au nombre d'ex æquo qui auront même durée fictive.

\section{Comparaison des méthodes et conclusion}

Une première comparaison des résultats obtenus est faite sur des échantillons connus dans la littérature sur ce sujet, en particulier avec les données de « Freireich» [7]. Les données de « Freireich » viennent d'un article de 1963 qui se rapporte à un essai thérapeutique (6-MP), il faut noter que ce jeu de données a été très utilisé dans la littérature concernant les études de données censurées et permet ainsi des comparaisons de méthodes.

Tableau comparatif des résultats obtenus avec les trois méthodes à partir de l'échantillon de Freireich (traitement $(6-\mathrm{MP}))$

- Synthèse des résultats comparatifs.

\begin{tabular}{lllll}
\hline Méthode utilisée & $\beta$ & $\eta$ & $\gamma$ & MTBF \\
\hline MJ & 0,688 & 31,99 & 5,984 & 17,095 \\
MDF $_{\text {MER }_{(2)}}^{0,939}$ & 12,91 & 5,702 & 17,095 \\
\hline
\end{tabular}

- Tableau complet des résultats comparatifs (Tab. 1). Une deuxième comparaison est faite à partir d'échantillons obtenus par simulation de Monte Carlo. Ces échantillons sont obtenus à partir d'un protocole de traitement défini afin d'éviter toutes mauvaises interprétations. Un nombre important d'échantillons a été simulé, ici nous ne présentons qu'un extrait de dix échantillons représentatifs de nos résultats en cohérence avec les autres. D'autre part un graphique de la fonction cumulée de défaillances est présenté sur un papier de Weibull pour une loi représentant les données simulées.

Les tableaux comparatifs des résultats obtenus par simulation sont les tableaux $2,3,4,5$. 
Tableau 2. Comparaisons de $b$ et $h$ avec $25 \%$ de censures.

\begin{tabular}{|c|c|c|c|c|c|c|c|c|}
\hline $\begin{array}{l}\text { No. de } \\
\text { l'éch. }\end{array}$ & $\begin{array}{l}\beta \text { avec les } \\
\text { données } \\
\text { de } \\
\text { références } \\
\text { et } 0 \% \text { de } \\
\text { censure }\end{array}$ & $\begin{array}{l}\beta \text { avec les } \\
\text { données } \\
\text { censurées } \\
\text { à } 25 \% \text { et } \\
\text { «FJ » } \\
\text { Johnson }\end{array}$ & $\begin{array}{l}\beta \text { avec les } \\
\text { données } \\
\text { censurées } \\
\text { à } 25 \% \text { et } \\
\text { « MDF » }\end{array}$ & $\begin{array}{l}\beta \text { avec les } \\
\text { données } \\
\text { censurées } \\
\text { à } 25 \% \text { et } \\
\text { « } \operatorname{MER}_{(2)} »\end{array}$ & $\begin{array}{l}\eta \text { avec les } \\
\text { données } \\
\text { de } \\
\text { références } \\
\text { et } 0 \% \text { de } \\
\text { censure }\end{array}$ & $\begin{array}{l}\eta \text { avec les } \\
\text { données } \\
\text { censurées } \\
\text { à } 25 \% \text { et } \\
\text { « FJ » } \\
\text { Johnson }\end{array}$ & $\begin{array}{l}\text { avec les } \\
\text { données } \\
\text { censurées } \\
\text { à } 25 \% \text { et } \\
\text { « MDF » }\end{array}$ & $\begin{array}{l}\eta \text { avec les } \\
\text { données } \\
\text { censurées } \\
\text { à } 25 \% \text { et } \\
\text { « } \operatorname{MER}_{(2)} \text { » }\end{array}$ \\
\hline 1 & 0,8103 & 0,8353 & 0,8676 & 0,822 & 79,755 & 108,70 & 76,934 & 112,04 \\
\hline 2 & 0,7732 & 0,8713 & 0,7915 & 0,9485 & 92,486 & 117,97 & 87,624 & 120,39 \\
\hline 3 & 0,7859 & 0,8417 & 0,8948 & 0,8662 & 117,30 & 140,55 & 104,25 & 140,27 \\
\hline 4 & 0,7405 & 0,6531 & 0,7438 & 0,585 & 60,403 & 67,209 & 51,179 & 64,312 \\
\hline 5 & 0,9314 & 0,9359 & 0,7985 & 0,8627 & 197,64 & 220,44 & 147,99 & 222,77 \\
\hline 6 & 0,7693 & 0,7216 & 0,7901 & 0,5963 & 81,684 & 103,20 & 75,968 & 112,56 \\
\hline 7 & 0,9732 & 0,9709 & 1,0011 & 0,9663 & 143,44 & 169,72 & 130,88 & 172,61 \\
\hline 8 & 0,6220 & 0,9146 & 0,7335 & 0,6331 & 124,20 & 198,73 & 122,99 & 177,51 \\
\hline 9 & 0,6882 & 0,6292 & 0,6712 & 0,6337 & 137,23 & 180,00 & 114,24 & 178,34 \\
\hline 10 & 0,9253 & 0,8654 & 0,8588 & 0,8574 & 111,71 & 146,64 & 99,839 & 150,14 \\
\hline
\end{tabular}

Tableau 3. Comparaisons de $g$ avec $25 \%$ de censures.

\begin{tabular}{|c|c|c|c|c|}
\hline $\begin{array}{l}\text { No. de } \\
\text { l'éch. }\end{array}$ & $\begin{array}{l}\gamma \text { avec les } \\
\text { données } \\
\text { de } \\
\text { références } \\
\text { et } 0 \% \text { de } \\
\text { censure }\end{array}$ & $\begin{array}{l}\gamma \text { avec les } \\
\text { données } \\
\text { censurées } \\
\text { à } 25 \% \text { et } \\
\text { «FJ » } \\
\text { Johnson }\end{array}$ & $\begin{array}{l}\gamma \text { avec les } \\
\text { données } \\
\text { censurées } \\
\text { à } 25 \% \text { et } \\
\text { « MDF » }\end{array}$ & $\begin{array}{l}\gamma \text { avec les } \\
\text { données } \\
\text { censurées } \\
\text { à } 25 \% \text { et } \\
\text { « } \operatorname{MER}_{(2)} »\end{array}$ \\
\hline 1 & 9,9773 & 9,9773 & 9,9773 & 9,9773 \\
\hline 2 & 9,7021 & 4,5270 & 4,5270 & 7,2156 \\
\hline 3 & 9,1158 & 7,5848 & 7,5848 & 7,1647 \\
\hline 4 & 10,2393 & 12,3572 & 9,9469 & 14,8495 \\
\hline 5 & 1,1337 & $-6,9602$ & 7,0271 & 7,0271 \\
\hline 6 & 8,8870 & 8,8114 & 8,8114 & 10,4711 \\
\hline 7 & 4,0361 & 4,0361 & 4,0361 & 4,0361 \\
\hline 8 & 10,2807 & $-13,4111$ & 3,7978 & 10,0608 \\
\hline 9 & 11,0983 & 11,3466 & 11,3466 & 11,3466 \\
\hline 10 & 9,8679 & 9,3866 & 11,0235 & 9,3866 \\
\hline
\end{tabular}

Tableau 4. Comparaisons de $b$ et $h$ avec $50 \%$ de censures.

\begin{tabular}{|c|c|c|c|c|c|c|c|c|}
\hline $\begin{array}{l}\text { No. de } \\
\text { l'éch. }\end{array}$ & $\begin{array}{l}\beta \text { avec les } \\
\text { données } \\
\text { de } \\
\text { références } \\
\text { et } 0 \% \text { de } \\
\text { censure }\end{array}$ & $\begin{array}{l}\beta \text { avec les } \\
\text { données } \\
\text { censurées } \\
\text { à } 50 \% \text { et } \\
\text { «FJ » } \\
\text { Johnson }\end{array}$ & $\begin{array}{l}\beta \text { avec les } \\
\text { données } \\
\text { censurées } \\
\text { à } 50 \% \text { et } \\
\text { « MDF » }\end{array}$ & $\begin{array}{l}\beta \text { avec les } \\
\text { données } \\
\text { censurées } \\
\text { à } 50 \% \text { et } \\
\text { « } \operatorname{MER}_{(2)} » \\
\text { censure }\end{array}$ & $\begin{array}{l}\eta \text { avec les } \\
\text { données } \\
\text { censurées } \\
\text { références } \\
\text { et } 0 \% \text { de } \\
\text { Johnson }\end{array}$ & $\begin{array}{l}\eta \text { avec les } \\
\text { données } \\
\text { de } \\
\text { à } 50 \% \text { et } \\
\text { « FJ » }\end{array}$ & $\begin{array}{l}\eta \text { avec les } \\
\text { données } \\
\text { censurées } \\
\text { à } 50 \% \text { et } \\
\text { « MDF » }\end{array}$ & $\begin{array}{l}\eta \text { avec les } \\
\text { données } \\
\text { censurées } \\
\text { à } 50 \% \text { et } \\
\text { « } \operatorname{MER}_{(2)} \text { » }\end{array}$ \\
\hline 1 & 0,8103 & 0,7021 & 0,7943 & 0,6807 & 79,755 & 105,84 & 51,739 & 111,25 \\
\hline 2 & 0,7732 & 0,881 & 0,8176 & 0,7829 & 92,486 & 195,01 & 84,514 & 191,42 \\
\hline 3 & 0,7859 & 0,8635 & 0,8040 & 0,8588 & 117,30 & 201,11 & 90,353 & 209,16 \\
\hline 4 & 0,7405 & 0,7643 & 0,6134 & 0,8406 & 60,403 & 97,202 & 39,509 & 101,72 \\
\hline 5 & 0,9314 & 1,0886 & 0,8889 & 0,8887 & 197,64 & 323,76 & 136,29 & 303,24 \\
\hline 6 & 0,7693 & 1,0485 & 0,7007 & 1,2531 & 81,684 & 125,130 & 52,656 & 130,51 \\
\hline 7 & 0,9732 & 1,0292 & 0,7391 & 1,2434 & 143,44 & 200,27 & 76,757 & 213,40 \\
\hline 8 & 0,6220 & 0,8212 & 0,6352 & 0,7984 & 124,20 & 320,18 & 108,75 & 343,43 \\
\hline 9 & 0,6882 & 0,6725 & 0,9145 & 0,5878 & 137,23 & 187,82 & 106,05 & 219,10 \\
\hline 10 & 0,9253 & 0,6691 & 1,1166 & 0,7280 & 111,71 & 192,74 & 115,43 & 215,72 \\
\hline
\end{tabular}


Tableau 5. Comparaisons de $g$ avec $50 \%$ de censures.

\begin{tabular}{|c|c|c|c|c|}
\hline $\begin{array}{l}\text { No. de } \\
\text { l'éch. }\end{array}$ & $\begin{array}{l}\gamma \text { avec les } \\
\text { données } \\
\text { de } \\
\text { références } \\
\text { et } 0 \% \text { de } \\
\text { censure }\end{array}$ & $\begin{array}{l}\gamma \text { avec les } \\
\text { données } \\
\text { censurées } \\
\text { à } 50 \% \text { et } \\
\text { «FJ» } \\
\text { Johnson }\end{array}$ & $\begin{array}{l}\gamma \text { avec les } \\
\text { données } \\
\text { censurées } \\
\text { à } 50 \% \text { et } \\
\text { « MDF » }\end{array}$ & $\begin{array}{l}\gamma \text { avec les } \\
\text { données } \\
\text { censurées } \\
\text { à } 50 \% \text { et } \\
\text { « } \operatorname{MER}_{(2)} »\end{array}$ \\
\hline 1 & 9,9773 & 14,8847 & 11,8504 & 16,9909 \\
\hline 2 & 9,7021 & $-5,7226$ & 7,9372 & 7,9372 \\
\hline 3 & 9,1158 & 6,6550 & 9,1166 & 6,6550 \\
\hline 4 & 10,2393 & 9,6735 & 7,0086 & 9,6735 \\
\hline 5 & 1,1337 & $-39,5316$ & $-1,6838$ & $-1,6838$ \\
\hline 6 & 8,8870 & 0 & 7,1387 & 0 \\
\hline 7 & 4,0361 & $-11,1784$ & 7,7768 & $-11,1784$ \\
\hline 8 & 10,2807 & $-1,6694$ & 10,7976 & $-1,6694$ \\
\hline 9 & 11,0983 & 15,001 & $-5,9844$ & 18,7327 \\
\hline 10 & 9,8679 & 18,2266 & 0 & 19,5557 \\
\hline
\end{tabular}

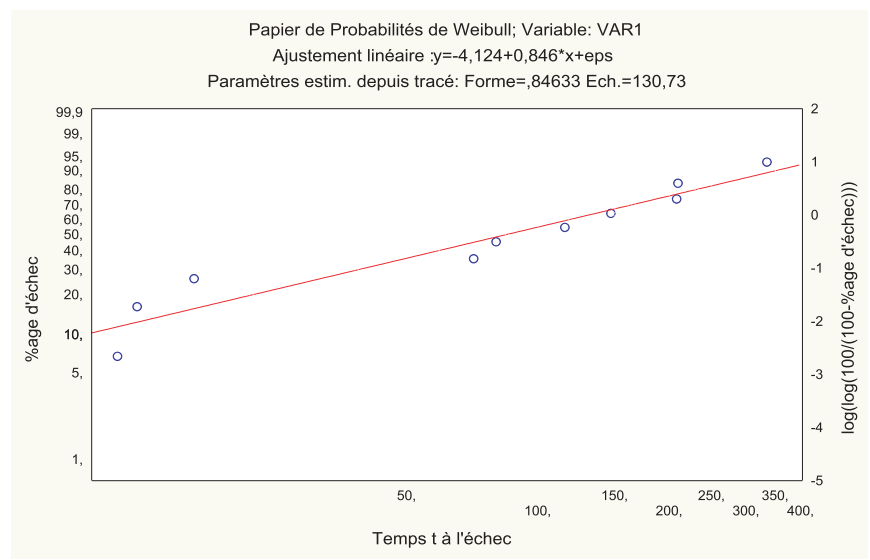

\section{Bilan, conclusion}

L'évaluation de la performance des méthodes est fait à partir d'un critère d'écart maximum obtenu par les trois solutions en compétitions, qui n'est pas détaillé ici afin de ne pas trop alourdir l'exposé. Nous constatons que lorsqu'il y a peu de censures (moins de $25 \%$ ) les trois méthodes donnent des résultats semblables (avec un léger avantage à la méthode «MDF), le paramètre « $\beta »$ est relativement bien obtenu, ce qui est rassurant compte tenu de son importance en fiabilité. Dans ce cas la méthode «FJ», plus facile à mettre en œuvre convient bien, les résultats au niveau du paramètre $« \beta »$ sont très bons. Par contre lorsque le taux de censures devient important (50\%), la méthode de Johnson conduit à des résultats plus erronés. La méthode des durées de vie fictive « $\mathrm{MDF}$ » donne de très bons résultats dans tous les cas et avec de fortes censures, ce qui est en particulier bien vérifié sur le paramètre d'échelle « $\eta$ », de ce fait, nous ne pouvons que la conseiller dans les applications industrielles à fort taux de censures.

\section{Références}

[1] P. Lyonnet, J. Fauchon, Comparaison de la méthode d'estimation des rangs dans le cas de données fortement censurées proposée par Johnson et de celle proposée par P.Lyonnet, Revue de Statistique appliquée XXXX (3) (1992) 73-78

[2] L. Johnson, The statistical treatment of fatigue experiment, Elsevier Publishing Company, 1964

[3] J.D. Kalbfleisch, R.L. Prentice, The statistical Analysis of Failure Time Data, John Wiley \& Sons, New York, 1980

[4] A. Lannoy, Celleux, Diebolt. Estimation des durées de vie fortement censurées, Edition Eyrolles

[5] P. Lyonnet Estimation de la fonction de répartition de défaillances à partir d'une itération loi uniforme/loi de Weibull Revue de Statistique Appliquée, XXXIX (2) 1991, pp. 59-68

[6] P. Lyonnet, J. Fauchon, G. Herbin, Nouvelle méthode d'estimation des paramètres de durées de vie dans les essais incomplets, $8^{\mathrm{e}}$ Colloque international de Fiabilité et Maintenabilité, Grenoble 1992

[7] E.O. Freireich, et al. The effect of 6-mercaptoptomine on the duration of steroid induced remission in acute leukemia, Blood 21, 1963, pp. 699-716 